\title{
Strategies for the Microcharacterization of Structural Defects in Wide Band Gap Materials Using Cathodoluminescence Microanalysis.
}

\author{
Marion A. Stevens-Kalceff
}

School of Physics, University of New South Wales, Sydney, 2052 NSW Australia.

Electron Microscope Unit, Analytical Centre, University of New South Wales, Sydney, 2052 NSW Australia.

Microstructural defects can result from impurities (e.g. substitutional and interstitial impurities, inclusions, etc.), and native imperfections (e.g. vacancies, self-interstitials, dislocations, inclusions etc.). The physical and chemical properties of materials are strongly influenced by their microstructural defects. Defects can also give insight into the processes associated with their generation and incorporation into the microstructure of the material. X-ray microanalysis allows the concentration and microscopic spatial distribution of the chemical constituents of a material to be quantitatively determined. Cathodoluminescence microanalysis (CL) gives insight into the relative concentration and microscopic spatial distribution of impurity and native structural defects in amorphous and crystalline luminescent materials including minerals, ceramics, oxides, semiconductors and wideband gap materials, etc. Characteristic X-ray emission is due to electronic transitions between inner core levels. The emitted X-ray lines are narrow, characteristic of the host atom and their energy is related to the atomic number by Moseley's Law. There is no similar general relationship that identifies CL emissions which can be narrow or broad depending on the associated defect. CL is due to electronic transitions across the band gap between the conduction and valence bands which results in the non-incandescent emission of light (photons).[1] Each CL emission is associated with a particular luminescent center, however determination of the origin of the emission is generally challenging, particularly with brad overlapping emissions. CL is associated with impurities and native defects and may be strongly influenced by electron beam parameters, temperature, strain, etc. Although the sensitivity of CL microanalysis of impurities is generally several orders of magnitude better than electron microprobe analysis, [1]quantitative CL microanalysis is not generally achievable because of the lack of a general explanation for the wide variety of possible types of luminescent centers and radiative and competitive non-radiative recombination mechanisms involved in CL processes. Despite these limitations, CL microanalysis provides high sensitivity sub-micron spatial resolution, detection of trace levels of impurities and imperfections in luminescent materials. CL microanalysis enables the in situ monitoring of preexisting and electron irradiation induced modification of the structure of a material. It also enables the post irradiation assessment of damage in a material following metamictization or exposure to energetic radiation or forces. The characterization of trace impurities and microstructural defects is important in science and technology and therefore a challenge for electron microanalysis.

The information available about the microstructure of a range of real world specimens will be presented including in particular a comparison of the modification to the defect structure of materials including as silicon dioxide $\left(\mathrm{SiO}_{2}\right)$, alumina $\left(\mathrm{Al}_{2} \mathrm{O}_{3}\right)$ and diamond following electron irradiation, laser irradiation and/or ion implantation. CL microanalysis can provide spatially resolved information about the lateral and depth distribution of pre-existing and irradiation induced structural defects. Depth resolved CL microanalysis enables the non destructive depth distribution of impurities and structural imperfections to be determined. For example CL allows the performance of 
laser optics to be assessed.[2] CL spectra from laser damage of silica shows an increase in CL emission associated with oxygen vacancy defects, with a higher concentration in the near-surface regions, and a monotonically decreasing concentration further below the surface.

Ion implantation is used in semiconductor device fabrication to produce structures on the nanoscale. CL allows the experimental determination of the ion induced damage within the implanted specimen. This is demonstrated in ion implanted diamond and ion implanted silicon dioxide. Ion implantation induces a vibronic band at $\sim 2.2 \mathrm{eV}$ in diamond, which is associated with an $\mathrm{N}-\mathrm{V}$ center. The N-V center is a nitrogen impurity associated with a carbon vacancy in the diamond structure. The depth distribution of this defect has been mapped and is strongly correlated with the SRIM calculations of the energy loss of the ions within the diamond microstructure. Following ion implantation a material is typically then annealed to remove the collisional ion induced damage defects, and restore the structural integrity of the host lattice. In particular the sensitivity of the CL technique to structural as well as impurity defects enables the assessment of the effectiveness of various annealing processes following ion implantation. Ion implantation into silica (amorphous silicon dioxide) produces residual damage in the implanted regions that typical thermal annealing processes do not completely remove.[3] For example a broad emission observed at $\sim 3.5 \mathrm{eV}$ is observed in ion implanted and/or annealed silica and is attributed to inhomogeneous broadening of various impurity defects incorporated into the ion damaged structure during thermal annealing. Inhomogeneous broadening results from local disorder in the host lattice at the defect site. Inhomogeneous broadening of CL emissions is particularly evident in CL spectra from amorphous/ amorphized materials due to the different local environments experienced by the defects located at slightly different sites.

CL of gallium Focused Ion Beam processed crystalline silicon dioxide (quartz) and crystalline alumina (sapphire) reveals oxygen associated vacancy associated defects in the CL spectra. The constituent elements are preferentially sputtered by the $30 \mathrm{keV}$ ion beam. In particular emissions associated with oxygen deficient defects in quartz observed at $\sim 2.7 \mathrm{eV}$ and $\sim 4.3 \mathrm{eV}$ are enhanced by focussed ion beam processing. Oxygen vacancy associated emission at $\sim 3 \mathrm{eV}$ is also enhanced from focussed ion beam processed sapphire. Sapphire is commonly used as substrate material in nanotechnology device applications.

In technology applications the microanalysis of materials provides information which allows the design and performance of a material/device to be monitored and iteratively optimized. CL microanalysis is a useful complementary method to X-ray microanalysis as it enables high sensitivity detection of structural defects as well as trace levels of impurities. Knowledge of the defect microstructure is essential to optimize device design and performance.

\section{References}

[1] Yacobi, B.G., \& Holt, D.B. (1990) Cathodoluminescence Microscopy of Inorganic Solids. Plenum Press, New York.

[2] Stevens-Kalceff, M.A., and Wong, J. (2005) Journal of Applied Physics, 97(11).

[3] Stevens-Kalceff, M.A., Prawer, S., Kalceff, W., Orwa, J.O., Peng, J.L., McCallum, J.C., and Jamieson, D.N. (2008) Journal of Applied Physics, 104(11), 113514-9.

[4] This research was supported by the Australian Research Council. Author contact: Marion.Stevens-Kalceff@unsw.edu.au 